\title{
Carbon Bonding Determination with XES Using a TES Microcalorimeter Detector
}

George J. Havrilla ${ }^{1}$, Kathryn McIntosh ${ }^{1}$, Mark Croce ${ }^{1}$, Rachel Huber ${ }^{1}$, David Podlesak ${ }^{1}$, Michael Rabin $^{1}$, Fernando Vila ${ }^{2}$, Matthew Carpenter ${ }^{3}$, Robin Cantor $^{3}$

${ }^{1}$ Los Alamos National Laboratory, New Mexico, USA

${ }^{2}$ University of Washington, Seattle, WA

${ }^{3}$ STAR Cryoelectronics, Santa Fe, NM

TES microcalorimeter detectors are capable of high-resolution X-ray emission spectroscopy (XES) which rivals XANES spectroscopic probes found only within the confines of synchrotrons. Commercial microcalorimeters offer spectral resolution around 5-7 eV which rivals that of wavelength dispersive XRF instruments yet provide full spectra of the material of interest not merely a single element, thereby surpassing WDXRF systems capabilities.

The bonding of carbon is predicated on the $\mathrm{sp}^{2} / \mathrm{sp}^{3}$ orbitals. By measuring the peak position of the carbon $\mathrm{K} \alpha$ peak around $282 \mathrm{eV}$ as well as the peak shape we can obtain information on the bonding of the carbon in the material under study.

We report the measurement of the carbon $\mathrm{K} \alpha$ peak for several materials of interest, amorphous carbon, nanodiamond and graphite since all three have different carbon forms and $\mathrm{sp} 2 / \mathrm{sp} 3$ bonding ratios. In addition, soot specimen from a contained detonation of explosive material was measured to determine the carbon species formed from the detonation. A high resolution microcalorimeter mounted on a scanning electron microscope was used to measure the carbon peak for these different materials. The powder samples were pressed into indium foil which was then placed onto a stub with carbon tape. In this manner, we eliminate the possibility of contaminating the spectra from the carbon tape background. The microcalorimeter detector has a resolution of around $5 \mathrm{eV}$ and 16 individual pixels each collecting a full spectrum. This microcalorimeter was used to detect the X-ray fluorescence emission of the four specimens using an electron beam at $5 \mathrm{kV}$ and beam current of $\sim 55 \mathrm{~mA}$. Full spectra from $\sim 100 \mathrm{eV}$ up to $\sim 3000 \mathrm{eV}$ were collected at $2 \mathrm{~min}$ intervals 5 times for a total accumulation time of 10 minutes. The individual spectra from each pixel were summed to obtain increased counting statistics for more accurate peak position measurements. In total, 60 spectra were collected for a total collection time of 120 minutes.

Figure 1 shows an overlay of a 2-minute acquisition of the carbon $\mathrm{K} \alpha$ spectra around $282 \mathrm{eV}$ for all the specimens. In examining these spectra, it is noted that the peaks are all downshifted from the expected $282 \mathrm{eV}$ position. Secondly, each peak has distinct peak positions and differences in peak shape indicating differences in the $\mathrm{sp}^{2} / \mathrm{sp}^{3}$ ratio for each material. A Gaussian fit to the peaks allows us to obtain the peak positions. If we use the amorphous carbon as the carbon reference point, the nanodiamond shows the largest shift towards higher energy relative to amorphous carbon. The graphite shows a small negative shift towards lower energy relative to amorphous carbon. The soot sample appears to be most similar to the amorphous carbon, but does exhibit a small positive shift indicating potential nanodiamond character.

Preliminary theoretical simulations of the XES of these materials using the transition potential approximation in density functional theory indicate similar trends in both the shape and position of the peaks, and support the experimental measurements. We used simple molecular models for the systems of interest that are representative of the different local environments found around the excited centers. This allows us to separate the different regions of each peak according to the source of its intensity. Moreover, 
the theoretical results predict a clear relation between the shift relative to the graphite peak and the local out of plane distortion around the absorbing center, a quantity that is closely related to its $\mathrm{sp} 2 / \mathrm{sp} 3$ character.

$\mathrm{X}$-ray photoelectron spectroscopy provides the $\mathrm{sp}^{2} / \mathrm{sp}^{3}$ hybridizations through bombardment of the sample surface with $\mathrm{x}$-rays that cause the subsequent release of electrons with the characteristic bonding energy of carbon: $\mathrm{sp}^{2}$ at $284 \mathrm{eV}$ and $\mathrm{sp}^{3}$ at $285 \mathrm{eV}$. Graphite samples shows purely $\mathrm{sp}^{2}$ character, where nanodiamond shows a mixture of $\mathrm{sp}^{2} / \mathrm{sp}^{3}(26 \% / 39 \%)$. The soot sample is a majority $67 \%$ sp ${ }^{2}$ with $16 \%$ $\mathrm{sp}^{3}$ character. Raman spectroscopy provides further information as to the $\mathrm{sp}^{2}$ allotropes and surface functionalization. For example with the soot we have defect $\mathrm{sp}^{2}$ carbon and amorphous carbon but the surface is not extensively oxygenated.

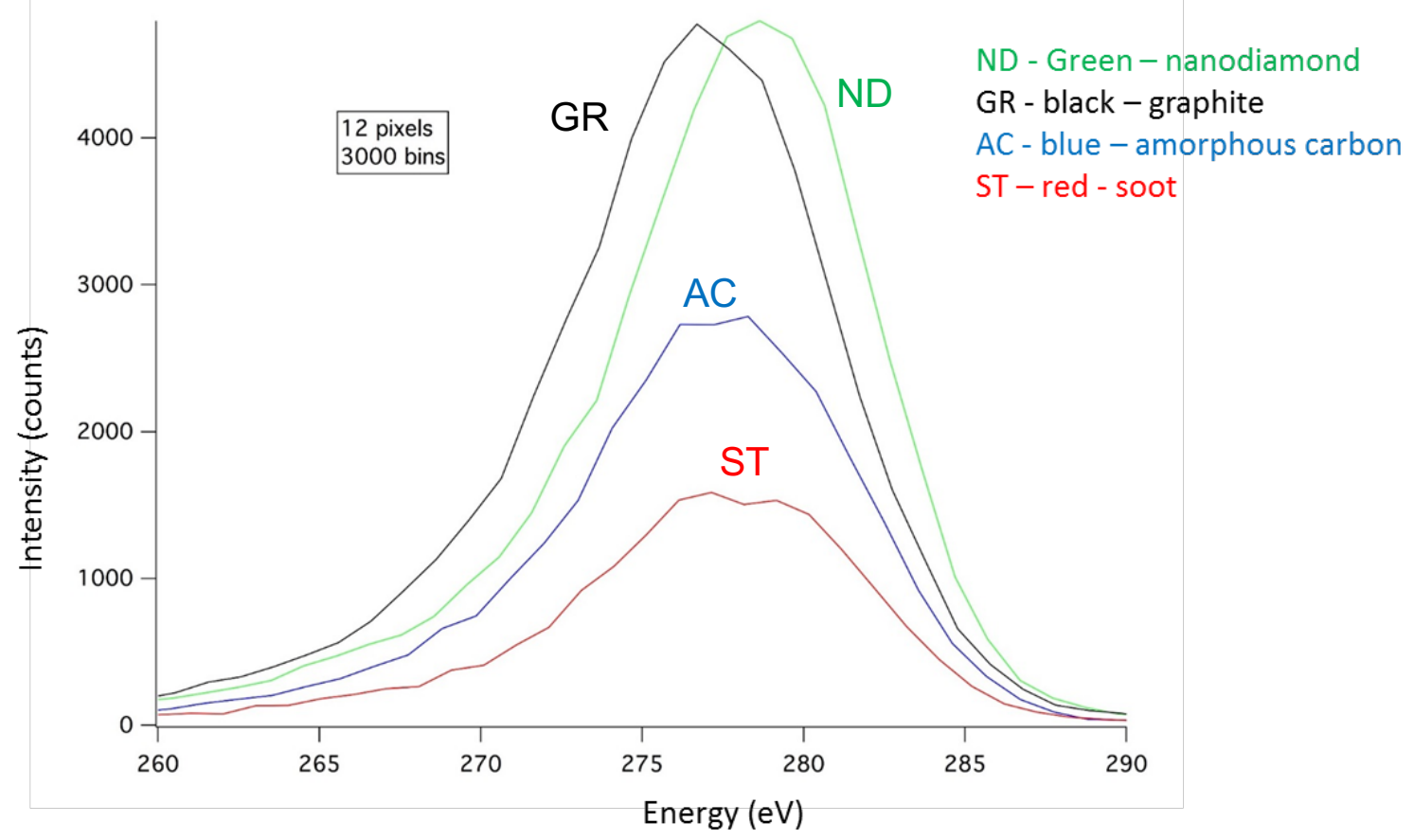

Figure 1. SEM XES spectra overlay of the carbon Ka peak for nanodiamond, graphite, amorphous carbon and soot. These spectra are from 12 pixels of a TES microcalorimeter acquired for 2 minutes for each material. 